\title{
Tolérance au déficit hydrique de cinq espèces prioritaires pour le reboisement au Burkina Faso
}

\author{
François Wenemi Kagambèga ${ }^{(1)}$, Rasmata Nana ${ }^{(2)}$, Philippe Bayen ${ }^{(3)}$, \\ Adjima Thiombiano ${ }^{(3)}$, Joseph Issaka Boussim ${ }^{(3)}$
}

(1) Centre National de Recherche Scientifique et Technologique. Institut de l'Environnement et de Recherches Agricoles. Département Environnement et Forêt. 03 BP 70247. Ouagadougou 03 (Burkina Faso). E-mail : wenemifrancois@yahoo.fr (2) Université Ouaga 1 Pr Joseph Ki-Zerbo. Unité de Formation et de Recherche en Sciences de la Vie et de la Terre. Laboratoire Biosciences/Équipe d'Écophysiologie Végétale. 03 BP 7021. Ouagadougou 03 (Burkina Faso).

(3) Université Ouaga 1 Pr Joseph Ki-Zerbo. Unité de Formation et de Recherche en Sciences de la Vie et de la Terre. Laboratoire de Biologie et Écologie Végétale. 03 BP 7021. Ouagadougou 03 (Burkina Faso).

Reçu le 8 octobre 2018, accepté le 3 septembre 2019, mis en ligne le 22 octobre 2019.

Cet article est distribué suivant les termes et les conditions de la licence CC-BY (http://creativecommons.org/licenses/by/4.0/ deed.fr)

Description du sujet. Dans les régions arides et semi-arides, la disponibilité en eau est l'un des facteurs limitant de la croissance et du développement des arbres. L'étude de la réponse au déficit hydrique des espèces végétales permettrait de sélectionner du matériel végétal résistant pouvant se développer dans les zones sujettes à des déficits hydriques périodiques.

Objectifs. L'objectif est d'évaluer la tolérance au stress hydrique de cinq espèces prioritaires pour le reboisement que sont Faidherbia albida, Acacia senegal, Jatropha curcas, Combretum micranthum et Pterocarpus lucens.

Méthode. L'induction du stress a consisté à soumettre des jeunes plants d'un mois à divers régimes d'arrosage combinant deux facteurs : la quantité d'eau apportée $(100 \%, 50 \%$ et $25 \%$ de la réserve utile du substrat de sol utilisé) et la fréquence d'arrosage ( 3 jours, 7 jours et 10 jours), à travers un dispositif complètement randomisé avec 10 traitements hydriques, dans un essai sous serre. Les performances de croissance et les indices de tolérance des plants soumis au stress ont été mesurés pour chaque traitement.

Résultats. Les résultats montrent qu'à l'exception de Pterocarpus lucens, caractérisée par de très faibles indices de tolérance en condition de stress sévère, les quatre autres essences sont performantes du fait de leur faible sensibilité au déficit hydrique et de leur taux de survie élevé. L'espèce Acacia senegal s'est révélée plus tolérante au déficit hydrique, suivie respectivement de Jatropha curcas, Combretum micranthum et Faidherbia albida.

Conclusions. Les plants de ces quatre espèces constituent donc un matériel prometteur pour le reboisement des milieux soumis fréquemment à des déficits hydriques, comme les zippela.

Mots-clés. Tolérance à la sécheresse, terres dégradées, plantes ligneuses, reconstitution forestière.

\section{Water stress tolerance potential of five priority species for afforestation in Burkina Faso}

Description of the subject. In arid and semi-arid regions, water availability is among the limiting factors that compromise the growth and development of tree species. The study of the response to water stress of plant species can lead to the selection of resistant plant material, which may be capable of becoming established in areas subject to periodic water deficits.

Objectives. This paper aimed to assess the tolerance to water stress of five priority species for afforestation: Faidherbia albida, Acacia senegal, Jatropha curcas, Combretum micranthum, and Pterocarpus lucens.

Method. The stress experiment consisted of subjecting one-month-old plants to various watering regimes combining two factors: water quantity provided $(100 \%, 50 \%$, and $25 \%$ of water-holding capacity of the substrate) and watering frequency (3 days, 7 days, and 10 days), through a completely randomized design with 10 treatments, in a greenhouse trial. Growth performance and stress tolerance indices of plants were monitored in each treatment.

Results. Results showed that, except for Pterocarpus lucens, which was characterized by very low tolerance indexes under severe stress conditions, the other selected species appeared efficient because of their low sensitivity to water deficit and their high survival rates. Acacia senegal appeared the most tolerant to water deficit, followed by Jatropha curcas, Combretum micranthum, and Faidherbia albida. 
Conclusions. Seedlings of these last four species could provide promising material for afforestation operations in environments frequently subjected to water deficits, such as zippela.

Keywords. Drought tolerance, degraded land, woody plants, reforestation.

\section{INTRODUCTION}

La dégradation des terres, qui se traduit par une perte de la productivité des sols, met en péril les moyens de subsistance d'un milliard de personnes dans le monde (CNULCD, 2015). Au stade ultime de la dégradation, les terres sont dénudées, quasi imperméables et stériles, appelées zippela en langue locale mooré au Burkina Faso (Mando et al., 1999). Les zippela sont caractérisés par leur forte compacité et leur faible niveau de fertilité (Ambouta et al., 1996 ; Kagambèga et al., 2011). Ainsi, la régénération de la végétation est quasi-absente à cause des mauvaises conditions hydriques et nutritives des sols. La restauration écologique et la réhabilitation s'inscrivent dans les recours possibles tant pour limiter l'extension des méfaits de la dégradation que pour en effacer ou en atténuer les conséquences (SER, 2002). Ainsi, ces méthodes de récupération du sol prônent le développement d'un couvert végétal, succession primaire et chainon indispensable à l'initiation du processus de restauration à travers une afforestation (Beggy \& Fehmi, 2016; Lu et al., 2018).

En zone soudano-sahélienne, les précipitations, par leur faiblesse et leur forte variabilité spatio-temporelle, constituent la principale contrainte à l'afforestation (Larwanou et al., 2010). L'adéquation entre les espèces ligneuses et les conditions des sites est également l'un des facteurs déterminants de réussite dans les opérations de reboisement (Gebrehiwot et al., 2005). Aussi, l'autre défi à relever est-il d'intégrer la science avec les connaissances endogènes dans le souci d'améliorer de manière durable les conditions de vie et de production des ménages. Ainsi, depuis quelques années, des travaux de recherche ont été conduits au Sahel afin d'intégrer les connaissances paysannes dans le choix des espèces et des technologies utilisées par les paysans dans la gestion de l'environnement et l'amélioration de leurs conditions de vie (Larwanou, 2013). Sur la base de ces travaux, un certain nombre d'espèces ligneuses prioritaires ont été définies. Certaines de ces espèces à forte valeur socio-économique, identifiées au Burkina Faso, sont Combretum micranthum, Pterocarpus lucens, Jatropha curcas, Acacia senegal et Faidherbia albida. Ces espèces sont appréciées par les populations rurales pour leurs divers services (pharmacologique, alimentaire, bioénergétique, écosystémique, etc.). Toutefois, la méconnaissance des caractéristiques biologiques et écologiques de ces espèces, et de leur adaptation aux stress abiotiques, limite leur utilisation adéquate (Gebrekirstos et al., 2006 ; Epila et al., 2017), surtout sur des types de terre sujets à des déficits hydriques périodiques, comme les zippela. Aussi, le succès des opérations d'afforestation se fonde-t-il sur la possibilité de recourir à du matériel végétal présentant encore toute l'étendue de sa diversité adaptative aux conditions de stress et de perturbations (Montwé et al., 2016 ; Boubacar et al., 2018).

Dans les pays sahéliens en général et au Burkina Faso en particulier, très peu d'informations sont disponibles sur la régénération et la croissance des essences forestières dans les conditions de déficit hydrique. Néanmoins, différents travaux démontrent que le déficit hydrique constitue le principal facteur qui réduit significativement le déroulement optimal de la germination des semences forestières (Dirik, 2000 ; Jaouadi et al., 2013). Toutefois, connaitre la réponse des espèces ligneuses aux contraintes hydriques est d'un grand intérêt pour la restauration écologique des terrains dégradés, en l'occurrence les zippela. Ces recherches sont nécessaires si l'on veut mieux comprendre les problèmes cruciaux de régénération observés dans ces milieux. En effet, la disponibilité en eau au niveau des sols est l'un des principaux facteurs limitant la croissance, la composition et la distribution des espèces d'arbres et arbustes (Reynolds et al., 2004 ; Otieno et al., 2005). Le déficit hydrique se traduit chez la plante par une série de modifications qui touchent les caractères morphologiques, physiologiques et biochimiques, à partir du moment où les besoins en eau de la plante sont supérieurs aux quantités disponibles (Mefti et al., 2001). La tolérance est cet état qui permet à la plante d'assurer normalement ses fonctions physiologiques malgré une dégradation de son état hydrique interne conséquente à la sècheresse (De Raïssac, 1992). La compréhension de la réponse des espèces vis-à-vis d'un stress hydrique ainsi que la caractérisation de leur portée de tolérance à ce stress sont nécessaires. Aussi, l'identification des caractères de tolérance à la sècheresse peut-elle permettre de sélectionner du matériel végétal résistant, pouvant croître et donner des rendements satisfaisants dans les zones sujettes à des déficits hydriques périodiques. C'est dans cette perspective que se situe la présente recherche dont l'objectif général est de répondre à la question suivante : les cinq espèces considérées sontelles potentiellement capables de tolérer un déficit hydrique pour croître de manière satisfaisante sur les zippela ? Les objectifs spécifiques de l'étude sont :

- déterminer les caractères d'adaptation au déficit hydrique des espèces étudiées ;

- évaluer leur résistance et leur portée de tolérance en cas de stress sévère. 
La connaissance de la réponse des espèces aux contraintes hydriques servirait de base pour proposer du matériel végétal adapté pour la réhabilitation des terres dégradées par afforestation. Les hypothèses suivantes ont été formulées :

- l'hétérogénéité temporelle de l'arrosage induit un stress hydrique qui affecte la croissance et le développement des plants des espèces étudiées ;

- les plants de ces espèces sont capables de tolérer le déficit hydrique imposé ;

- le niveau de tolérance au déficit hydrique diffère suivant les espèces.

\section{MATÉRIEL ET MÉTHODES}

\subsection{Matériel utilisé}

L'expérimentation a été conduite dans la serre de l'Unité de Formation et de Recherche en Sciences de la Vie et de la Terre, dont les coordonnées GPS sont $12^{\circ} 37^{\prime}$ de latitude nord et $01^{\circ} 49^{\prime}$ de longitude ouest. Les graines utilisées pour la production des plants ont été fournies par le Centre National des Semences Forestières du Burkina Faso ; elles ont été récoltées dans des localités précises du pays sur des peuplements naturels pour chacune des espèces étudiées, puis conservées dans une enceinte régulée à une température de $20{ }^{\circ} \mathrm{C}$. Les plants ont été cultivés dans des pots en sachets plastiques, sous une serre faite de clôture en grillage fin et la toiture, de tôles translucides. Un substrat composé d'un mélange de sable, de terre et de fumier de petits ruminants dans les proportions volumiques respectives de $1: 2: 1$ a été utilisé. Les semences de cinq espèces ont été semées dans des pots en plastique troués (mesurant $20 \mathrm{~cm} \times 30 \mathrm{~cm}$ à vide et à plat) qui, remplis du substrat, prennent une forme cylindrique avec un volume de $2600 \mathrm{~cm}^{3}$ chacun. Les semis ont été effectués à raison de deux graines par pot à une profondeur de $5 \mathrm{~cm}$. Deux semaines après les semis, il a été réalisé un démariage pour obtenir un plant par pot. Le substrat a été maintenu humide durant toute la période de production, les plantules se développant dans des conditions identiques jusqu'à la fin de la production.

\subsection{Conditions d'expérimentation}

Caractéristiques du substrat. Les résultats de l'analyse du substrat (Tableau 1) révèlent qu'il est de texture limono-sableuse $(10,25 \%$ d'argile, $47,81 \%$ de limon et $41,96 \%$ de sable) et dispose d'une quantité suffisante de nutriments (matière organique, azote, phosphore et potassium) pour permettre une croissance adéquate des plants si l'eau est disponible. Ce type de substrat a été choisi pour sa bonne capacité de rétention en eau et également parce qu'il permet au système racinaire d'absorber facilement l'eau qu'il contient (Nana et al., 2010). Dans un sol, il est également important de caractériser la capacité de rétention en eau et la réserve utile (RU) qui est la quantité d'eau disponible pour les arbres cumulée sur toute la profondeur d'enracinement (Bréda et al., 2002). Dans la présente étude, la RU a été déduite de la différence entre les teneurs à la capacité au champ $(\mathrm{pF} 2,5)$ et au point de flétrissement permanent $(\mathrm{pF} 4,2)$ après analyse du substrat (Tableau 1), afin de mieux catégoriser les niveaux d'induction du stress (Combres et al., 1999 ; Badeau \& Bréda, 2008).

Dispositif expérimental. À la suite de la production des plants, les pots contenant les plants des cinq espèces ont été aléatoirement répartis en 10 entités de 50 plants, dont 10 plants par espèce, à travers un dispositif complètement randomisé pour l'induction de stress. Ainsi, un mois après la levée, pendant que l'une de ces entités (témoin) est traitée dans les mêmes conditions que la période de production des plants, les neufs autres ont été soumises à un stress de déficit hydrique modéré à sévère, en régulant la quantité d'eau et la fréquence d'arrosage jusqu'à la fin de l'expérience. L'induction du stress a consisté en la soumission des plants à divers régimes d'arrosage combinant deux facteurs : la quantité d'eau à trois niveaux relatifs à la réserve utile (RU) du substrat de sol utilisé $(100 \%, 50 \%$ et $25 \%$ ) et la fréquence d'arrosage (trois jours, sept jours et dix jours). Au total, 10 traitements hydriques ont été définis comme suit :

- T0 : traitement témoin qui a consisté à un arrosage régulier chaque jour à $100 \%$ de la RU (100/1) jusqu'à la fin de l'expérience (alimentation hydrique non limitante);

Tableau 1. Caractéristiques physico-chimiques du substrat de sol utilisé pour l'expérience (moyenne \pm écart type ; $\mathrm{n}=$ 3) - Physicochemical characteristics of the soil substrate used for the experiment (mean \pm standard deviation; $n=3$ ).

\begin{tabular}{ll}
\hline Paramètre & Quantité \\
\hline Propriétés physiques & \\
Argile $(\%)$ & $10,25 \pm 0,65$ \\
Limons $(\%)$ & $47,81 \pm 0,06$ \\
Sables $(\%)$ & $41,96 \pm 0,09$ \\
Densité apparente $\left(\mathrm{g} \cdot \mathrm{cm}^{-3}\right)$ & $41,96 \pm 0,09$ \\
$\mathrm{pF} 2,5(\%)$ & $18,11 \pm 1,77$ \\
$\mathrm{pF} 4,2(\%)$ & $0,624 \pm 0,017$ \\
Propriétés chimiques & \\
Matière organique totale $\left(\mathrm{g} \cdot \mathrm{kg}^{-1}\right)$ & $16,6 \pm 0,43$ \\
Carbone total $\left(\mathrm{g} \cdot \mathrm{kg}^{-1}\right)$ & $9,05 \pm 0,21$ \\
Azote total $\left(\mathrm{g} \cdot \mathrm{kg}^{-1}\right)$ & $0,65 \pm 0,07$ \\
Phosphore $\left(\mathrm{cmol} \cdot \mathrm{kg}^{-1}\right)$ & $56,95 \pm 6,01$ \\
Potassium $\left(\mathrm{cmol} \cdot \mathrm{kg}^{-1}\right)$ & $42,2 \pm 1,27$ \\
$\mathrm{pH}\left(\mathrm{H}_{2} \mathrm{O}\right)$ & $6,43 \pm 1,2$ \\
\hline
\end{tabular}


- 100/3 : arrosage régulier tous les 3 jours à $100 \%$ de la RU jusqu'à la fin de l'expérience ;

- 100/7 : arrosage régulier tous les 7 jours à $100 \%$ de la RU jusqu'à la fin de l'expérience ;

- 100/10 : arrosage régulier tous les 10 jours à $100 \%$ de la RU jusqu'à la fin de l'expérience ;

- 50/3 : arrosage régulier tous les 3 jours à $50 \%$ de la RU jusqu'à la fin de l'expérience ;

- 50/7 : arrosage régulier tous les 7 jours à $50 \%$ de la RU jusqu'à la fin de l'expérience ;

- 50/10 : arrosage régulier tous les 10 jours à $50 \%$ de la RU jusqu'à la fin de l'expérience ;

- 25/3 : arrosage régulier tous les 3 jours à $25 \%$ de la

RU jusqu'à la fin de l'expérience ;

- 25/7 : arrosage régulier tous les 7 jours à $25 \%$ de la

RU jusqu'à la fin de l'expérience ;

- 25/10 : arrosage régulier tous les 10 jours à $25 \%$ de la RU jusqu'à la fin de l'expérience.

La durée de ces traitements hydrique a été de 60 jours.

En considérant les taux moyens d'humidité par jour $(\mathrm{Hm})$ et les fréquences d'arrosage, ces traitements ont été ordonnés du témoin au traitement à stress sévère comme suit : T0 ; 100/3;50/3 ; $100 / 7 ; 100 / 10 ; 25 / 3 ; 50 / 7 ; 50 / 10 ; 25 / 7$ et $25 / 10$ (Tableau 2).

Le seuil hydrique en dessous duquel les plantes se retrouvent en situation de déficit hydrique étant à
$40 \%$ de la réserve utile (Bréda \& Badeau, 2008), les traitements ont été regroupés en trois gammes :

- traitements sans déficit hydrique (T0 et 100/3) avec une humidité relative moyenne $(\mathrm{Hm})$ supérieure à $40 \%$ de la $\mathrm{RU}(\mathrm{Hm}>2,89 \%)$;

- traitements induisant un déficit hydrique modéré (50/3, 100/7, 100/10,25/3 et 50/7) avec une humidité relative moyenne comprise entre $10 \%$ et $40 \%$ de la RU $(0,7 \% \leq \mathrm{Hm}<2,89 \%)$;

- traitements induisant un déficit hydrique sévère $(50 / 10,25 / 7$ et $25 / 10)$ avec une humidité relative moyenne inférieure à $10 \%$ de la RU $(\mathrm{Hm}<0,7 \%)$.

Humidité relative et température. Compte tenu de l'influence de la température et de l'humidité relative de l'air sur le bilan hydrique, la croissance et le développement des plantes, un thermo-hygromètre portable à sonde intégrée de marque ARC-AR837 a été placé dans la serre pour relever quotidiennement à différentes heures de la journée $(9 \mathrm{~h}, 12 \mathrm{~h}$ et $17 \mathrm{~h})$ ces deux paramètres suscités. Au cours de l'expérimentation, l'humidité relative et la température moyenne journalière dans la serre ont évolué en dents de scie (Figure 1). Cette variation a été plus accentuée du début jusqu'au milieu de l'expérimentation, avec une humidité relative moyenne journalière parfois inférieure à $40 \%$ et des températures moyennes journalières avoisinant $35^{\circ} \mathrm{C}$ avec une légère hausse par moment. Par la suite, les températures se sont

Tableau 2. Taux moyens d'humidité du sol $(\mathrm{Hm})$ des différents traitements hydriques au début de l'expérience - Average soil moisture levels $(\mathrm{Hm})$ in the different water treatments at the beginning of the trial.

\begin{tabular}{lllllllllll}
\hline & \multicolumn{1}{l}{ Traitements } \\
\cline { 2 - 11 } & T0 & $\mathbf{1 0 0 / 3}$ & $\mathbf{5 0 / 3}$ & $\mathbf{1 0 0 / 7}$ & $\mathbf{1 0 0 / \mathbf { 1 0 }}$ & $\mathbf{2 5 / 3}$ & $\mathbf{5 0 / 7}$ & $\mathbf{2 5 / 7}$ & $\mathbf{5 0 / 1 0}$ & $\mathbf{2 5 / 1 0}$ \\
\hline $\operatorname{Hm}(\%)$ & 11,54 & 3,84 & 1,92 & 1,64 & 1,15 & 0,96 & 0,82 & 0,58 & 0,41 & 0,29 \\
\hline
\end{tabular}

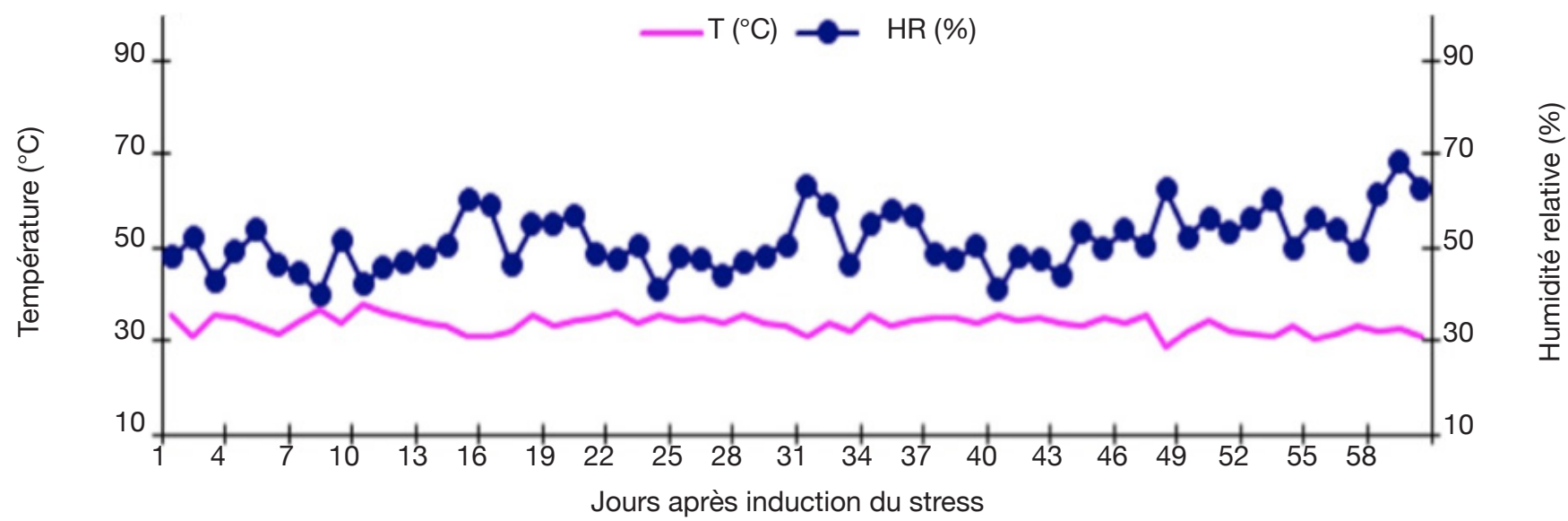

Figure 1. Évolution de la température (T) et de l'humidité relative moyenne (HR) journalière dans la serre au cours de la période d'induction du stress - Evolution of daily temperature $(T)$ and average relative humidity $(H R)$ in the greenhouse during the stress induction period. 
relativement stabilisées autour de $30^{\circ} \mathrm{C}$, avec toutefois de légères baisses par moment ; l'humidité relative, quant à elle, a varié entre $40 \%$ et $70 \%$ pendant toute la période d'induction de déficit hydrique. En faisant la relation entre la température et l'humidité relative moyenne hors serre dans la ville de Ouagadougou et celles relevées dans la serre en avril-juin, on constate qu'elles sont relativement comparables. Il en ressort que les conditions climatiques ne diffèrent pas significativement tout au long de l'expérimentation, en dehors de la courte période en fin d'expérimentation avec des températures moyennes inférieures à $30^{\circ} \mathrm{C}$ et une humidité relative supérieure à $70 \%$.

\subsection{Paramètres mesurés}

Paramètres morphophysiologiques. Le suivi de la croissance des plants a débuté le $30^{\mathrm{e}}$ jour après les semis et a continué régulièrement à une fréquence de 7 jours jusqu'à la fin de l'expérimentation. Ce suivi a consisté en des mesures de croissance verticale et radiale des plants, et en un comptage du nombre de feuilles produites par plant. Les mesures de croissance verticale et radiale ont consisté respectivement en la mesure de la hauteur et du diamètre au collet. La hauteur de chaque plant, exprimée en centimètre, a été mesurée à l'aide d'un ruban et le diamètre au collet de la tige, exprimée en millimètre, a été mesuré à l'aide d'un pied à coulisse. Toutefois, ce sont les données de la fin de l'expérience qui ont été utilisées dans ce présent travail pour caractériser les effets des traitements hydriques sur les plants.

À la fin de l'expérience, des échantillons de cinq plants par traitement et par espèce ont été prélevés pour l'analyse de certaines caractéristiques morphologiques, à savoir : la longueur de la racine $(\mathrm{cm})$, le poids sec de la partie aérienne végétative $(\mathrm{g})$ et le poids sec de la partie racinaire $(\mathrm{g})$. Les poids secs ont été déterminés après séchage dans une étuve à $65^{\circ} \mathrm{C}$ pendant $72 \mathrm{~h}$ à l'aide d'une balance de précision $(0,01 \mathrm{~g})$. Les racines ont été prélevées après élimination du sol par trempage dans l'eau. Ces paramètres permettent de mieux comprendre le mécanisme adopté par chaque espèce dans les conditions de déficit hydrique et d'évaluer ses potentialités d'adaptation. Le rapport racines/tiges est étudié comme moyen d'adaptation pour limiter les pertes d'eau.

Indices de performances. Les indices de performance des espèces, tels le taux de survie et l'indice de sensibilité à la sècheresse (ISS), ont été calculés à la fin de l'expérience suivant les différents traitements ; cela en vue de caractériser et de comparer les degrés de tolérance des différentes espèces au déficit hydrique.

Le taux de survie (TS) dans les différents traitements est calculé pour chaque espèce par la formule suivante :

$$
\operatorname{TS}(\%)=100 \times \frac{N_{0}-N_{v}}{N_{0}}
$$

avec $\mathrm{N}_{0}$, le nombre de plantes au début de l'expérience (soit 10 pour tous les traitements) et $\mathrm{N}_{\mathrm{v}}$, le nombre de plantes vivantes à la fin de l'expérience.

Dans cette étude, l'indice de sensibilité à la sècheresse, mis en évidence par Fischer et Maurer (1978) et déjà utilisé avec succès dans de nombreuses études (Bahar \& Yildirim, 2010 ; Nana et al., 2010), a été déterminé à partir de deux paramètres séparément (la hauteur des plants et la longueur de leur racine), par la formule ci-dessous :

$$
\operatorname{ISS}(\%)=100 \times \frac{P M T-P M S}{P M T}
$$

avec PMT, les valeurs des paramètres mesurés (hauteur des plants et longueur de la racine) sur les plantes témoins et PMS, celles des paramètres mesurés sur les plantes stressées.

Ainsi, les indices de sensibilité à la sècheresse à partir de la hauteur des plants (ISS-H) et à partir de la longueur de la racine (ISS-R) ont été déterminés pour chaque espèce suivant les différents traitements.

\subsection{Traitement des données}

Pour apprécier les différences entre les traitements hydriques au niveau de chaque espèce, les données obtenues à partir des paramètres mesurés ont fait l'objet d'une analyse de variance et, à chaque fois qu'il existe des différences significatives, les moyennes ont été séparées et classées par le test Tukey HSD (Honestly Significant Difference) au seuil de $5 \%$. Une analyse de variance à deux facteurs a été utilisée pour évaluer les différences de performance (ISS et TS) entre les espèces en réponse aux traitements hydriques ; les effets principaux et les effets d'interaction ont été évalués avec la procédure General Linear Models avec des erreurs de type binomial. Le modèle linéaire généralisé avec des erreurs de type binomial a été utilisé pour pallier la distribution non-normale des erreurs et l'inconstance de la variance qui sont associées aux données binaires. Toutes les analyses statistiques ont été réalisées avec le logiciel JMP 12 (SAS Institute Inc, 2015).

\section{RÉSULTATS}

\subsection{Effet du déficit hydrique sur les paramètres morphophysiologiques}

L'effet provoqué par le déficit hydrique a été généralement la réduction de la croissance végétative 
(Figure 2, Tableau 3). L'analyse de variance a révélé que les valeurs de hauteur $(\mathrm{dl}=9 ; \mathrm{F}=16,66 ; p<0,0001)$, de diamètre $(\mathrm{dl}=9 ; \mathrm{F}=5,13 ; p<0,0001)$ et de nombre de feuilles $(\mathrm{dl}=9 ; \mathrm{F}=11,51 ; p<0,0001)$ des plants sont significativement influencées par les traitements hydriques chez toutes les cinq espèces. Les valeurs de hauteurs sont plus élevées chez le témoin (T0) suivi du traitement 100/3, puis les traitements à déficit modéré, et plus faibles chez les plants des traitements à stress hydrique sévère. Les tendances notées pour la hauteur des plants sont similaires à celles observées pour le diamètre, de même que pour le nombre de feuilles et la biomasse sèche, pour toutes les espèces. Toutefois, il est à signaler que les valeurs de hauteur, de diamètre et de biomasse ont été en général plus élevées chez J. curcas, suivi par celles de C.micranthum, A. senegal, $F$. albida et les plus faibles valeurs sont notées chez $P$. lucens ; le nombre de feuilles est plus élevé chez $F$. albida suivi par A. senegal, puis $C$. micranthum, $J$. curcas et plus faible chez P. lucens. Cependant, pour ce qui est du ratio racine/tige, hormis $C$. micranthum, $F$. albida et $P$. lucens, l'effet des traitements n'est pas significativement différent chez $A$. senegal $(\mathrm{dl}=9 ; \mathrm{F}=$ $2,399 ; p=0,059)$ et chez $J$. curcas $(\mathrm{dl}=9 ; \mathrm{F}=0,892 ; p=$ $0,549)$. Chez ces dernières, les valeurs sont plus élevées pour A. senegal et faibles chez J. curcas, indiquant ainsi un développement racinaire plus important chez $A$. senegal. Pour les espèces $C$. micranthum, $F$. albida et $P$. lucens, où des différences significatives ont été observées suivant les traitements hydriques, le classement des moyennes montre des valeurs plus élevées dans les traitements à déficit hydrique sévère et modéré (à l'exception des traitements $25 / 3$ et $50 / 3$ ) et plus faibles dans les traitements sans contrainte hydrique (T0 et 100/3) ; cela indique une augmentation significative de la longueur de la racine en rapport avec la taille des plants sous l'effet du stress hydrique chez ces trois espèces. Les résultats de la biomasse sèche montrent également chez ces trois dernières espèces des rapports (biomasse racinaire/biomasse aérienne) plus élevés dans les traitements à déficit hydrique sévère par rapport aux traitements sans déficit hydrique (Tableau 3).

\subsection{Effets des traitements hydriques sur les indices de performance}

Les résultats obtenus montrent une variabilité importante de l'indice de sensibilité à la sècheresse (ISS), ainsi que du taux de survie, chez les espèces étudiées sous l'influence des traitements appliqués (Figure 3). En effet, l'analyse de variance à deux voies montre une variation significative de ces paramètres en fonction des traitements hydriques et des espèces étudiées (Tableau 4). Les indices de sensibilité (ISS$\mathrm{H}$ et ISS-R, respectivement à partir de la hauteur des

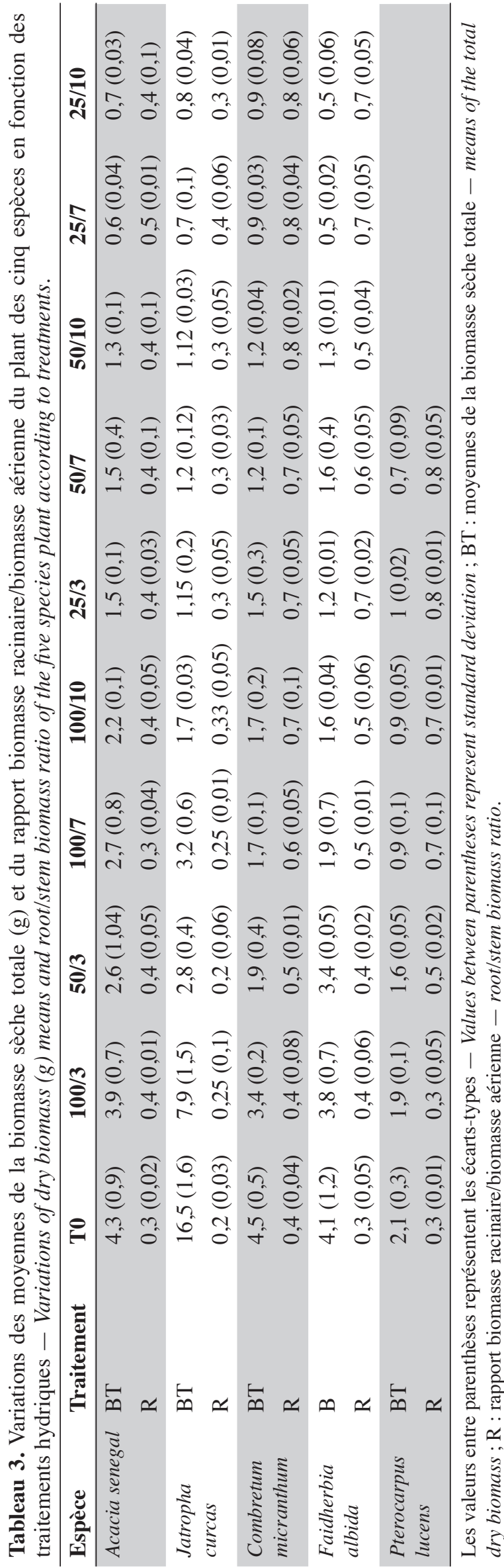


Pterocarpus lucens
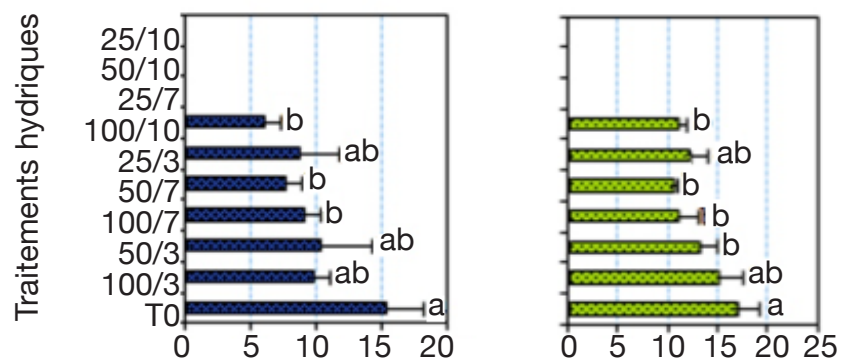

Faidherbia albida

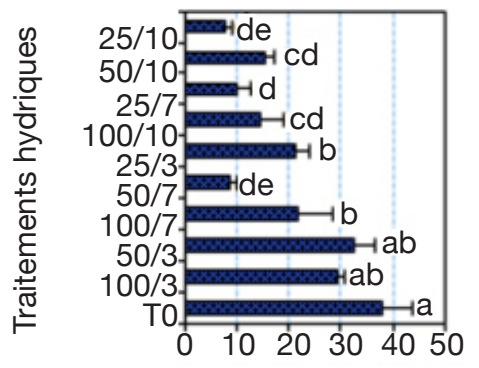

\section{Combretum micranthum}

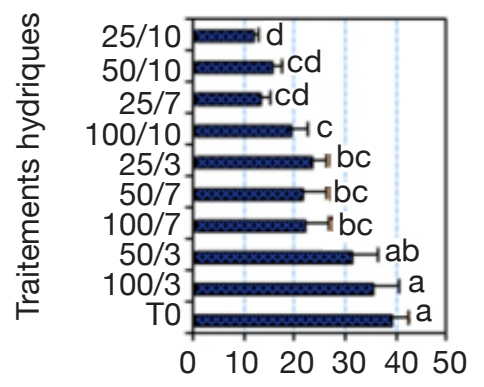

Jatropha curcas

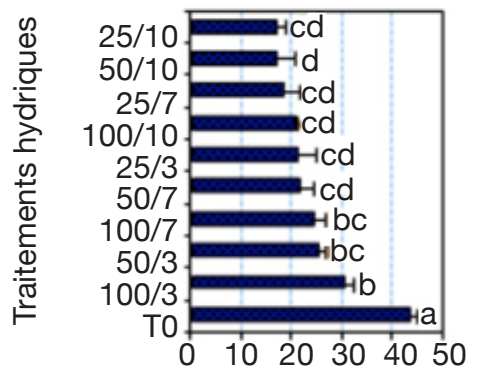

Acacia senegal

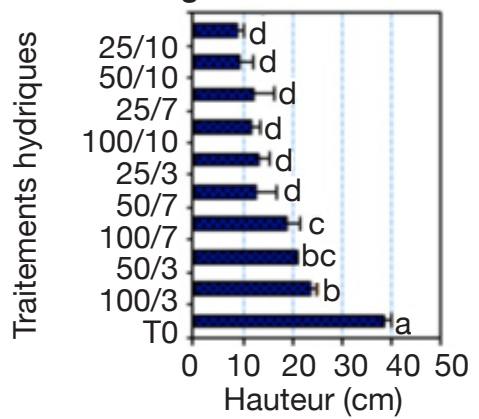

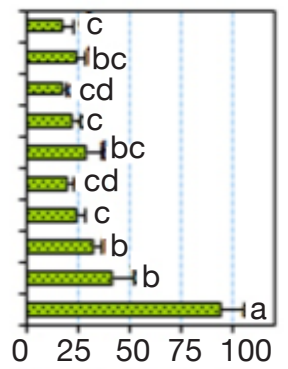
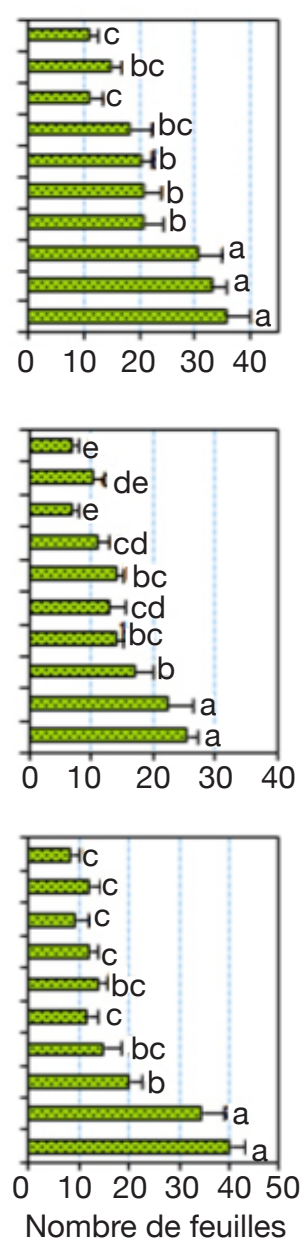
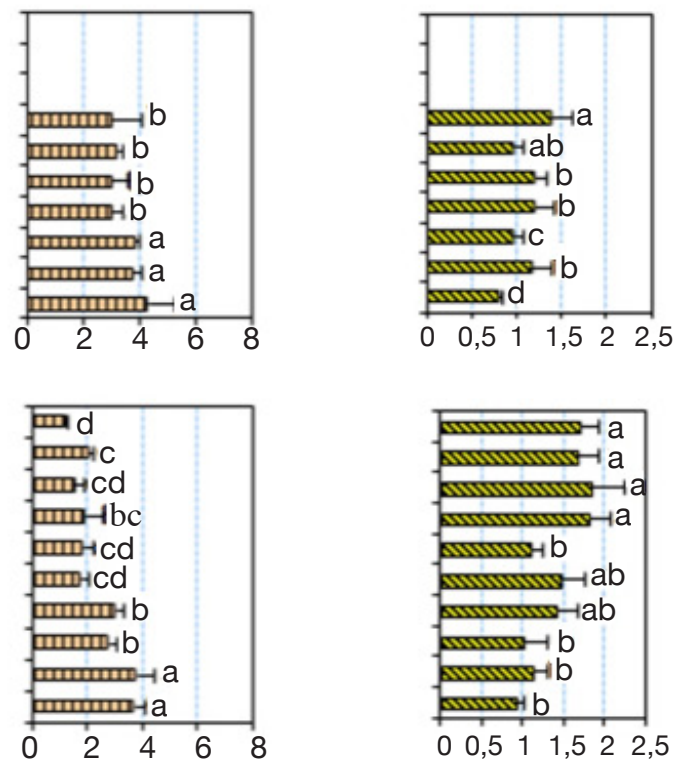

$00,511,522,5$
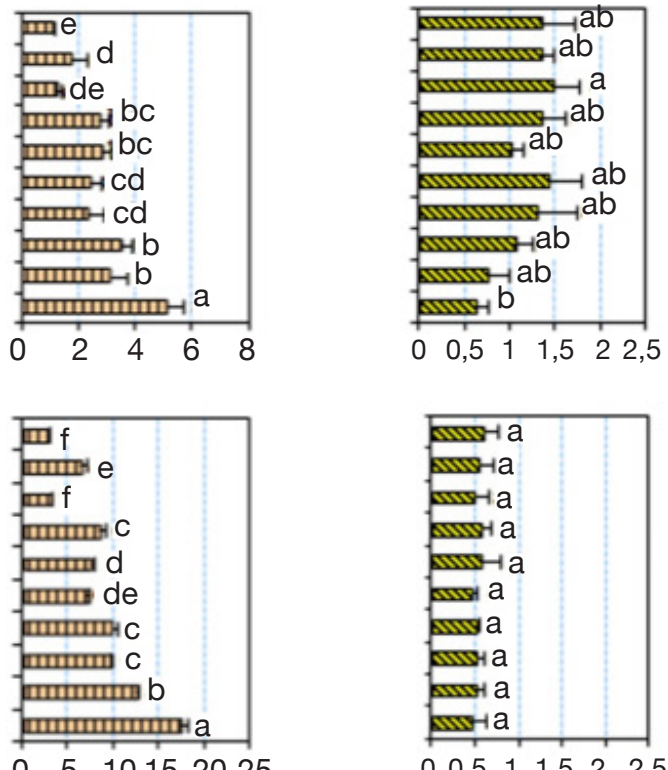

$0 \quad 510152025$

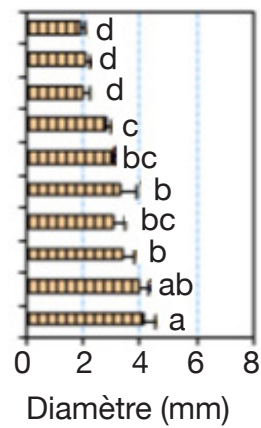

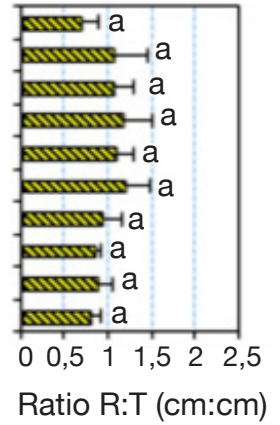

Figure 2. Variations des paramètres morphophysiologiques (hauteur, nombre de feuilles et ratio racine/tige) des cinq espèces en fonction des traitements hydriques - Variations of morphophysiological parameters (height, number of leaves and root/ stem ratio) of the five species according to the water treatments.

Les valeurs utilisées sont des moyennes et les lettres dénotent la significativité au seuil de $5 \%$ selon le test Tukey HSD - Values are means and the different letters denote significant differences according to Tukey's HSD test ; Les barres sont des écarts-types - The error bars represent standard deviation. 


\section{Pterocarpus lucens}
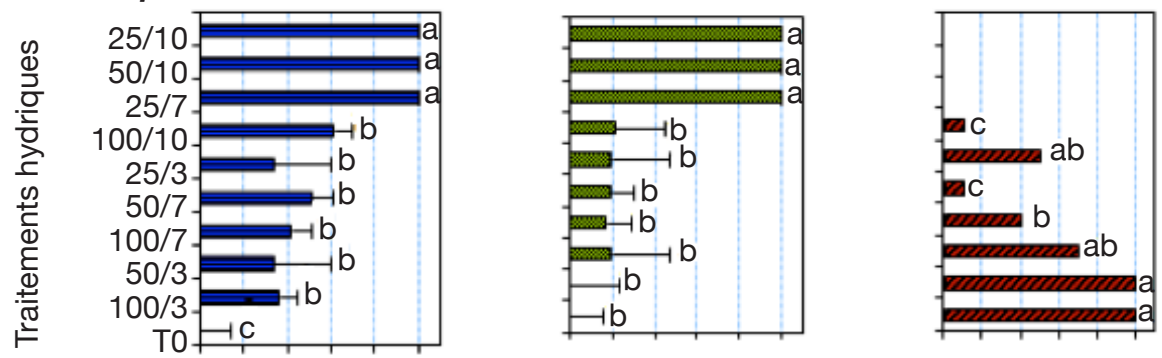

\section{Faidherbia albida}
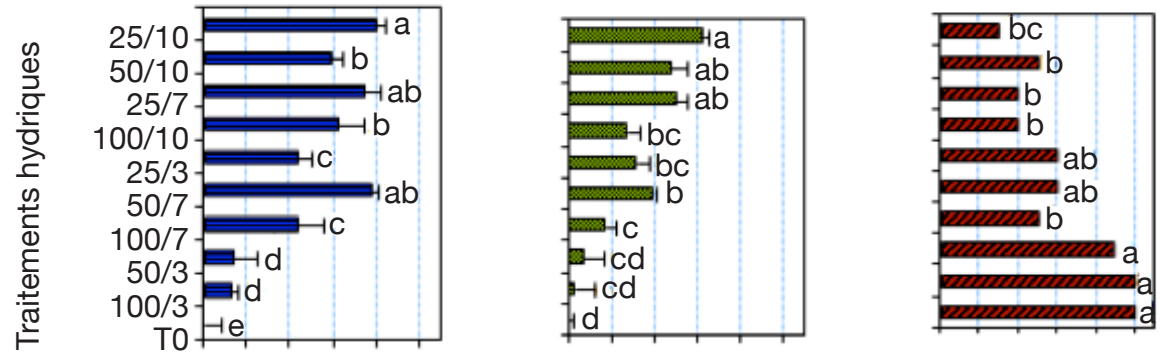

\section{Combretum micranthum}

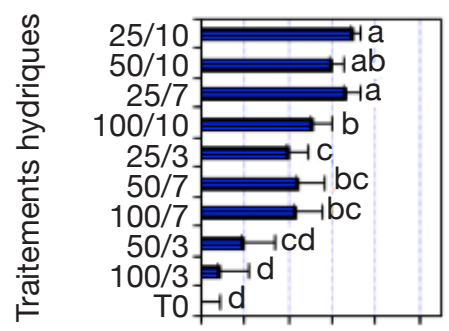

\section{Jatropha curcas}
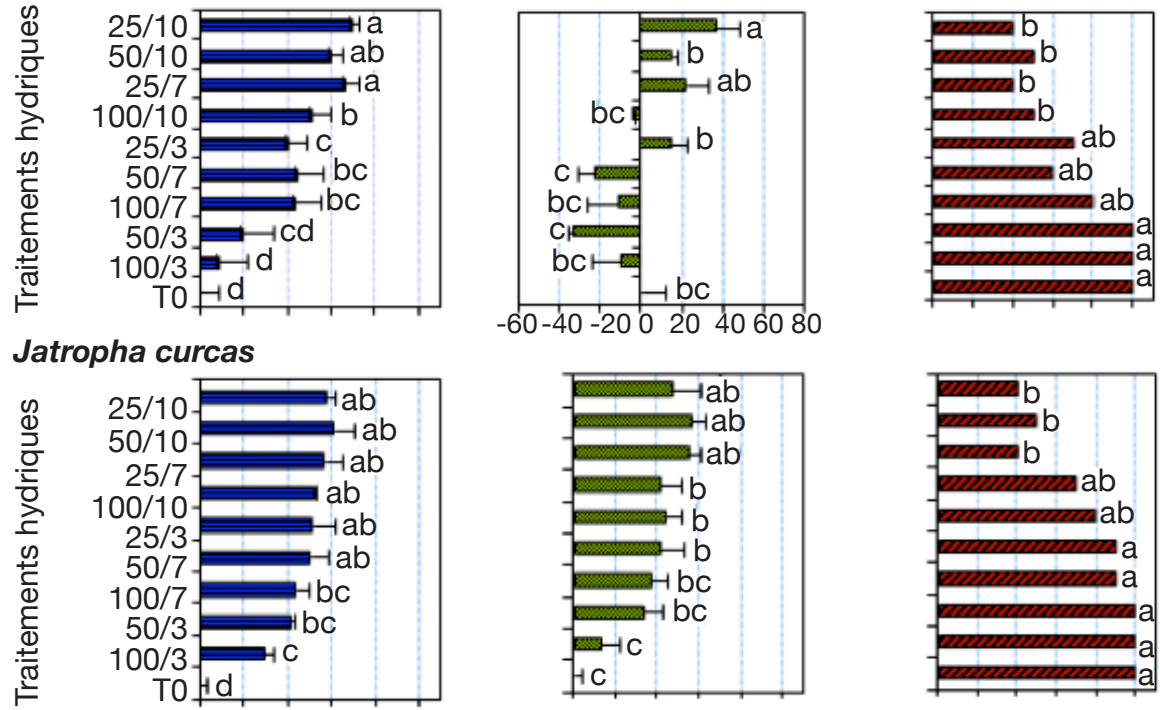

\section{Acacia senegal}
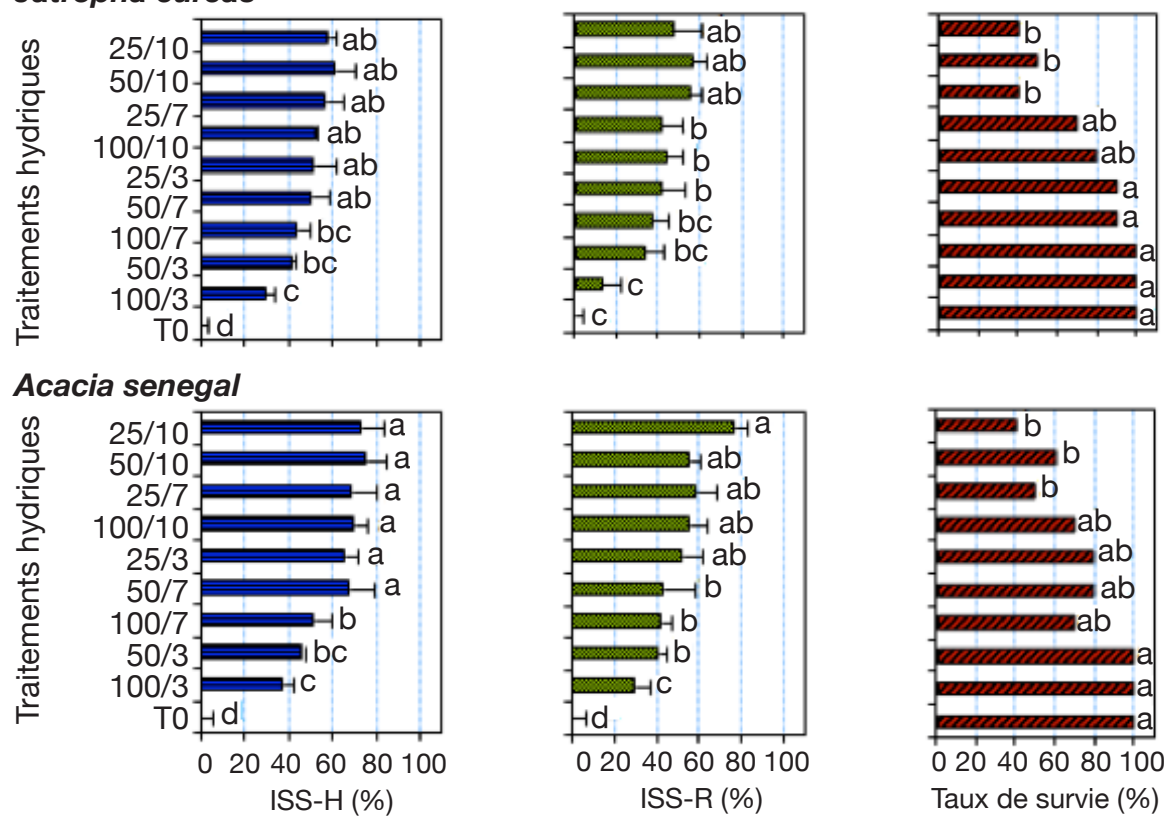

Figure 3. Variations des indices de sensibilité au stress hydrique et du taux de survie des espèces suivant les traitements hydriques - Variations in drought sensitivity indices and survival rates of species following water treatments.

Les valeurs utilisées sont des moyennes et les lettres dénotent la significativité au seuil de $5 \%$ selon le test Tukey HSD - Values are means and the different letters denote significant differences according to Tukey's HSD test ; Les barres sont des écartstypes - error bars represent standard deviation; ISS-H : indices de sensibilité au stress à partir de la hauteur des plants — stress sensitivity index from plants height; ISS-R : indices de résistance au stress à partir de l'élongation de la racine - stress sensitivity index from the elongation of the roots. 
Tableau 4. Résultats de l'analyse de variance avec les indices de sensibilité à la sècheresse à la fin de l'essai comme variables dépendantes, le type d'espèce et le traitement comme facteurs fixes - Results of analysis of variance with sensitivity index at the end of the trial as dependent variables, species and treatment as fixed factors.

\begin{tabular}{|c|c|c|c|c|c|c|}
\hline \multirow[t]{2}{*}{ Source de variation } & \multicolumn{3}{|c|}{ ISS-H } & \multicolumn{3}{|c|}{ ISS-R } \\
\hline & dl & $\mathbf{F}$ & $p$ & dl & $\mathbf{F}$ & $p$ \\
\hline Espèce & 4 & 17,53 & $<0,0001 *$ & 4 & 64,85 & $<0,0001 *$ \\
\hline Traitement & 9 & 102,97 & $<0,0001 *$ & 9 & 50,03 & $<0,0001^{*}$ \\
\hline Espèces $\times$ Traitement & 36 & 3,91 & $<0,0001 *$ & 36 & 5,54 & $<0,0001^{*}$ \\
\hline
\end{tabular}

*: les moyennes sont significativement différentes - means are significantly different ; ISS-H : indice de sensibilité à la sècheresse à partir de la hauteur des plants — drought sensitivity index from plants height; ISS-R : indice de sensibilité à la sècheresse à partir de l'élongation de la racine - drought sensitivity index from the elongation of the roots.

plantes et de la longueur de la racine principale) augmentent progressivement lorsqu'on évolue des traitements sans déficit hydrique aux traitements à déficit hydrique sévère en passant par ceux à déficit hydrique modéré chez toutes les espèces, à l'exception de $C$. micranthum pour l'ISS-R où les traitements à stress modéré induisent des indices moins élevés que ceux du traitement sans déficit hydrique. Ces résultats indiquent qu'en situation de déficit hydrique, ces espèces réagissent en réduisant leur croissance en hauteur et leur élongation racinaire par rapport aux plantes témoins, sauf chez $C$. micranthum où l'élongation racinaire était plus importante dans les traitements à déficit hydrique modéré. Les taux de survie (TS) ont également varié suivant les espèces sous l'influence des traitements. Contrairement aux ISS, ce paramètre a diminué progressivement des traitements sans déficit hydrique $(100 \%)$ aux traitements à stress hydrique sévère (entre $0 \%$ et $80 \%$ ), en passant par les traitements à stress hydrique modéré (entre $45 \%$ et $80 \%$ ). Toutefois, l'effet du déficit hydrique sur ce paramètre a été plus marqué chez $P$. lucens avec un taux de survie nul dans les traitements à stress hydrique sévère.

En considérant uniquement les traitements à stress hydrique sévère $(50 / 10 ; 25 / 7$ et $25 / 10)$, l'analyse de variance montre une variation hautement significative de l'IRS ( $\mathrm{dl}=4 ; \mathrm{F}=17,28$ et $p<0,0001)$, de l'ISS-H (dl $=4 ; \mathrm{F}=41,82$ et $p<0,0001)$ et des taux de survie $(\mathrm{dl}=4 ; \mathrm{F}=90,75$ et $p<0,0001)$ suivant les espèces étudiées. Sous ces conditions, la sensibilité à la sècheresse estimée à partir de la hauteur des plants a varié significativement suivant les espèces, ainsi que les taux de survie (Figure 4). Les espèces qui ont été plus sensibles au déficit hydrique sont par ordre croissant $P$. lucens, suivi par A. senegal, $F$. albida, $C$. micranthum puis $J$. curcas. Les tendances notées sur les taux de survie des espèces ont montré que les meilleurs taux de survie ont été notés chez A. senegal et $J$. curcas, suivis respectivement par $C$. micranthum, F. albida puis $P$. lucens. En considérant l'ensemble des paramètres évalués, l'espèce $A$. senegal s'est montrée plus performante, suivie respectivement par J. curcas, C. micranthum et F. albida. Pterocarpus lucens apparait comme l'espèce la moins performante avec des plants très sensibles au déficit hydrique, qui n'arrivent pas à survivre dans les traitements à stress hydrique sévère.

\section{DISCUSSION}

Outre son rôle dans la photosynthèse, le transport et l'accumulation des éléments nutritifs, ainsi que dans

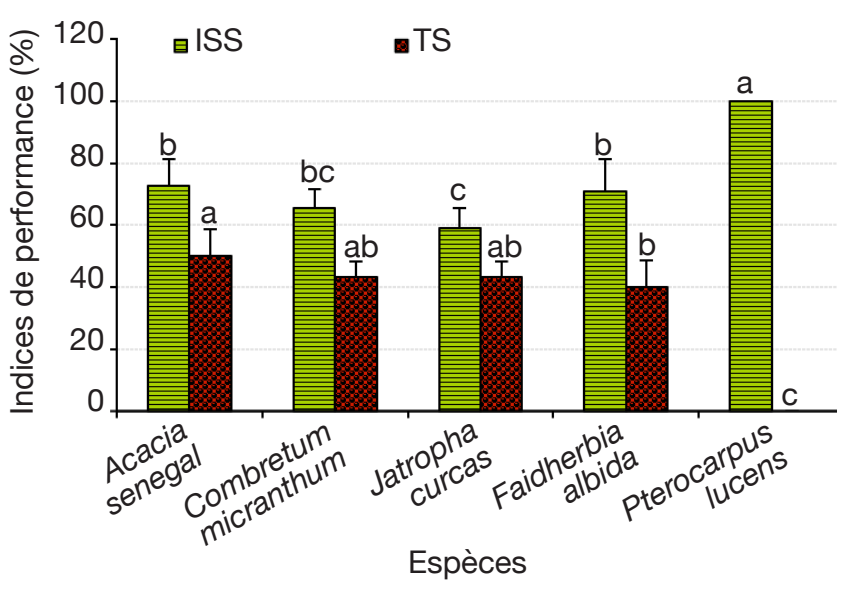

Figure 4. Performance des cinq espèces en fin d'expérimentation dans les traitements à stress hydrique sévère - Performance of the five species at the end of the experiment under the treatments with severe water stress.

ISS : indice de sensibilité à la sècheresse à partir de la hauteur des plants - drought sensitivity index from the height of the plants ; TS : taux de survie - survival rate; Les valeurs utilisées sont des moyennes et les lettres dénotent la significativité au seuil de $5 \%$ selon le test Tukey HSD - The values are means and the different letters denote significant differences according to Tukey's HSD test; Les barres sont des écarts-types - Error bars represent standard deviation. 
la régulation thermique, l'eau joue un rôle essentiel dans la croissance et le développement des plantes (Reynolds et al., 2004). La croissance est l'ensemble des changements quantitatifs irréversibles des organes qui se produisent au cours du temps. Elle implique à la fois la croissance et le développement cellulaire où les processus impliqués (division, différenciation et élargissement cellulaire) sont très sensibles au déficit hydrique en raison de leur dépendance à la turgescence (Ferreira et al., 2015). Un déficit en eau a généralement des effets réducteurs sur la croissance des plantes avec pour conséquence une baisse du rendement (Chaves, 2002). Les résultats de la figure 2 et du tableau 3 indiquent que les paramètres morphophysiologiques des plantes ont été nettement et négativement affectés par la restriction hydrique. Cette affection négative des variables pourrait s'expliquer par l'état juvénile des plants (plants d'un mois) pendant l'induction du déficit hydrique. En effet, la réponse d'une plante au manque d'eau est complexe et dépend à la fois du stade de développement de la plante, de la sévérité du stress, de la durée du stress et de l'état dans lequel se trouvait la plante quand le stress a eu lieu (Aziadekey et al., 2014). Chez toutes les espèces, la différence de performance a été en faveur des traitements témoins, suivi des traitements à déficit hydrique modéré puis les traitements à déficit sévère pour tous les paramètres, à l'exception du ratio racine-tige. Nos résultats corroborent ceux de Otieno et al. (2005) qui ont aussi constaté une diminution dans la croissance des plants sous l'effet du stress hydrique chez deux espèces du genre Acacia. En effet, le manque d'eau chez les plantes provoque une diminution du contenu relatif en eau et une réduction de la production, concomitante à une réduction de la croissance (Maréchaux et al., 2015). Chaves (2002) explique cette réduction de la croissance, suite à un déficit hydrique, comme un mécanisme de régulation permettant l'adaptation des plantes aux restrictions hydriques par une diminution de la surface transpirante. Cependant, il a été noté que les mesures se rapportant au ratio racine/tige sont totalement variables selon les espèces et suivant les traitements. L'analyse de la variance de ce paramètre a révélé une différence significative avec des valeurs plus élevées chez les plantes en condition de déficit hydrique, chez $C$. micranthum, $F$. albida et $P$. lucens, tandis qu'aucune différence par rapport au témoin n'a été notée chez A. senegal et J. curcas au seuil de $5 \%$. Les différences observées entre espèces seraient dues aux caractéristiques intrinsèques de chaque espèce, comme cela a été traduit par les indices de sensibilité (Figure 3). En effet, les plantes ligneuses ont une grande capacité et diversité de réponses aux stress hydriques (Hiernaux \& Le Houérou, 2006) et leur degré de tolérance au déficit hydrique varie selon les espèces et selon le stade de développement (Maréchaux et al., 2015). Les résultats indiquent que la réponse des racines et de la partie aérienne au déficit hydrique sont totalement différentes chez C. micranthum, F. albida et P. lucens, qui ont vraisemblablement un développement racinaire beaucoup plus important en condition de déficit hydrique dans le sol. La façon dont les plantes répondent aux stress nous permet de comprendre leur répartition géographique et leurs comportements en fonction de l'établissement de gradients des facteurs environnementaux (Hopkins \& Évrard, 2013). Au fur et à mesure que le niveau de stress s'intensifie, la dépression de la croissance racinaire est généralement moins importante que la dépression de la croissance aérienne (Chloupek et al., 2010). Ces résultats sont en accord avec ceux obtenus par Zgallaï et al. (2007) qui ont rapporté que sous déficit hydrique, la croissance des racines est moins inhibée que celle de la partie aérienne. Un système racinaire capable d'extraire l'eau du sol en situation de déficit hydrique est un critère essentiel pour la tolérance à la sècheresse (Chloupek et al., 2010). Ainsi, C. micranthum, F. albida et $P$. lucens ont réagi par un développement racinaire plus important par rapport à la partie aérienne dans les traitements en condition de stress hydrique, cela afin d'amoindrir les pertes d'eau par réduction de la partie transpirante, mais aussi d'augmenter l'absorption d'eau dans le sol. Toutefois, il est apparu que l'élongation racinaire était plus importante dans les traitements à déficit hydrique modéré par rapport à celle dans les traitements sans déficit hydrique chez C. micranthum; ce caractère indique un enracinement profond des plants de $C$. micranthum, permettant de récolter l'eau en profondeur lorsque celle-ci est limitée dans les couches superficielles du sol. Pour ce qui est de $A$. senegal et $J$. curcas, un certain équilibre a été noté entre la partie aérienne et la partie souterraine des plantes, indépendamment du degré de déficit appliqué, avec toutefois des valeurs de ratio élevées chez A. senegal $(0,7$ à 1,1$)$, surtout dans les traitements à stress modérés et faibles chez $J$. curcas $(<0,5)$. Cela s'explique par des stratégies particulières adoptées par ces espèces pour contrecarrer les effets néfastes du déficit hydrique. Les valeurs similaires du ratio, indépendamment du traitement appliqué, indique que les réactions au déficit hydrique, au niveau des racines et des parties aériennes, sont proportionnelles chez ces deux espèces. Les valeurs élevées de ratio racine/tige notées au niveau des plants stressés de A. senegal indiquent toutefois une adaptation racinaire de l'espèce. Ceci serait un indice d'adaptation pour la survie de l'espèce dans les zones de sècheresse par augmentation du volume racinaire et par réduction de la surface transpirante (Iwata et al., 2013). Cependant, les faibles valeurs du ratio racine/tige chez J. curcas se traduisent par une croissance aérienne plus importante en situation de déficit hydrique, ce qui constituerait 
une forme d'adaptation osmotique de l'espèce. Ce résultat est conforme aux observations de Maes et al. (2009) qui ont aussi noté que J. curcas est une plante succulente avec un système racinaire peu profond au stade jeune. Aussi, selon Borchert (1994), la tolérance au déficit hydrique de $J$. curcas n'est pas d'éviter le stress à travers un meilleur accès à l'eau du sol, mais à travers l'utilisation des réserves d'eau de la tige. La baisse de la production de la biomasse chez les plantes stressées peut être justifiée par la réduction de la croissance chez ces dernières. Les espèces qui ont un indice de résistance élevé sont celles qui maintiennent à la fois leur capacité d'ajustement osmotique et de production au cours d'une phase de sècheresse (Buldgen, 1997). Une plante est tolérante lorsqu'elle est capable de maintenir son activité métabolique sous de faibles potentiels d'eau. De ce point de vue, les espèces A. senegal, J. curcas, C. micranthum et $F$. albida sont respectivement les plus tolérantes au déficit hydrique ; cela est confirmé par leurs faibles indices de sensibilité et leurs taux de survie élevés en condition de stress. Par contre, l'espèce P. lucens, caractérisée par des indices de sensibilités élevés et des taux de survie nuls, est la moins tolérante au déficit hydrique dans le sol.

\section{CONCLUSIONS}

Cette étude sur la réponse au déficit hydrique des plants de cinq espèces ligneuses a montré que :

- le déficit hydrique affecte négativement les paramètres morphophysiologiques des plants ;

- les espèces testées réagissent différemment au déficit hydrique ;

- l'espèce Acacia senegal est la plus tolérante au déficit hydrique et la plus performante ; ensuite viennent respectivement les espèces Jatropha curcas, Combretum micranthum et Faidherbia albida, qui se valent sensiblement en termes de tolérance à la sècheresse ;

- l'espèce Pterocarpus lucens est la moins résistante du fait de sa faible performance en termes de croissance et de survie des plants.

Ces résultats montrent que sur les cinq espèces, quatre (A. senegal, C. micranthum, J. curcas et F. albida) sont performantes pour résister au déficit hydrique. Elles seraient donc vraisemblablement capables de développer des mécanismes de survie qui répondent bien aux contraintes hydriques stationnelles des zippela, auxquelles elles seront susceptibles de faire face en cas d'afforestation. Malgré ces résultats prometteurs, il n'en demeure pas moins que la croissance et le développement de ces espèces soient ralentis par les conditions écologiques défavorables de l'habitat des zippela. Une étude de terrain serait donc nécessaire afin de vérifier les résultats de cette étude.

\section{Bibliographie}

Ambouta J.M.K., Valentin C. \& Laverdière M.R., 1996. Jachères et croûtes d'érosion au Sahel. Sécheresse, 7(4), 269-275.

Aziadekey M., Atayi A., Odah K. \& Magamana A.-E., 2014. Étude de l'influence du stress hydrique sur deux lignées de niébé. Eur. Sci.J., 10(30), 328-338.

Badeau V. \& Bréda N., 2008. Modélisation du bilan hydrique : étape clé de la détermination des paramètres et des variables d'entrée. RDV Techn. ONF hors-série, 4.

Bahar B. \& Yildirim M., 2010. Heat and drought resistances criteria in spring bread wheat: drought resistance parameters. Sci. Res. Essays, 5(13), 1742-1745.

Beggy H.M. \& Fehmi J.S., 2016. Effect of surface roughness and mulch on semi-arid revegetation success, soil chemistry and soil movement. Catena, 143, 215-220.

Borchert R., 1994. Soil and stem water storage determine phenology and distribution of tropical dry forest trees. Ecology, 75(5), 1437-1449.

Boubacar A.A., Douma S. \& Diouf A., 2018. Effets du stress hydrique et de la température sur la germination de quatre ligneux alimentaires prioritaires du Niger. Afr. Sci., 14(3), 28-41.

Bréda N., Lefèvre Y. \& Badeau V., 2002. Réservoir en eau des sols forestiers tempérés : spécificité et difficultés d'évaluation. Houille Blanche, 3, 24-32.

Bréda N. \& Badeau V., 2008. Forest tree responses to extreme drought and some biotic events: towards a selection according to hazard tolerance? CR Geosci., 340(9-10), 651-662.

Buldgen A., 1997. Croissance juvénile et comportement physiologique d'Andropogon gayanus Kunth var. bisquamulatus en conditions de stress hydrique. Biotechnol. Agron. Soc. Environ., 1(4), 278-294.

Chaves M.M., 2002. How plants cope with water stress in the field? Photosynthesis and growth. Ann. Bot., 89(7), 907-916.

Chloupek O. et al., 2010. Drought tolerance of barley varieties in relation to their root system size. Plant Breed., 129(6), 630-636.

CNULCD, 2015. Changement climatique et dégradation des terres : relier les connaissances aux enjeux. In : Actes de la $3^{e}$ Conférence Scientifique de la CNULCD, 9-12 mars 2015, Cancún, Mexique, 34.

Combres J.C., Mezo L. Le, Mete M. \& Bourjon B., 1999. Réserve utile et mesures d'humidité. Difficulté de calage des modèles de bilan hydrique. Agric. Dev., 24, 39-47.

De Raïssac M., 1992. Mécanismes d'adaptation à la sécheresse et maintien de la productivité des plantes cultivées. Agron. Trop., 46(1), 29-39. 
Dirik H., 2000. Effet du stress hydrique osmotique sur la germination des graines chez les provenances de Cèdre du Liban (Cedrus libani A. Rich.) d'origine Turque. Ann. For. Sci., 57(4), 361-367.

Epila J. et al., 2017. Plant measurements on African tropical Maesopsis eminii seedlings contradict pioneering water use behaviour. Environ. Exp. Bot., 135, 27-37.

Ferreira W.N., de Lacerda C.F., da Costa R.C. \& Medeiros Filho S., 2015.Effect of water stress on seedling growth in two species with different abundances: the importance of Stress Resistance Syndrome in seasonally dry tropical forest. Acta Bot. Brasilica, 29(3), 375-382.

Fischer R. \& Maurer R., 1978. Drought resistance in spring wheat cultivars. I. Grain yield responses. Aust. J. Agric. Res., 29(5), 897.

Gebrehiwot K., Muys B., Haile M. \& Mitloehner R., 2005. The use of plant water relations to characterize tree species and sites in the drylands of northern Ethiopia. J. Arid. Environ., 60(4), 581-592.

Gebrekirstos A., Teketay D., Fetene M. \& Mitlöhner R., 2006. Adaptation of five co-occurring tree and shrub species to water stress and its implication in restoration of degraded lands. For. Ecol. Manage., 229(1-3), 259 267.

Hiernaux P. \& Le Houérou H.N., 2006. Les parcours du Sahel. Sécheresse, 17(1-2), 51-71.

Hopkins W.G. \& Évrard C.-M., 2013. Physiologie végétale. Bruxelles : De Boeck.

Iwata S., Miyazawa Y., Fujii N. \& Takahashi H., 2013. MIZ1-regulated hydrotropism functions in the growth and survival of Arabidopsis thaliana under natural conditions. Ann. Bot., 112(1), 103-114.

Jaouadi W. et al., 2013. Effet des contraintes hydrique et saline sur la germination de trois espèces d'acacias en Tunisie. Rev. Écol., 68(2), 133-141.

Kagambèga F.W., Traoré S., Thiombiano A. \& Boussim J.I., 2011. Impact de trois techniques de restauration des sols sur la survie et la croissance de trois espèces ligneuses sur les «zipellés» au Burkina Faso. Int. J. Biol. Chem. Sci., 5(3), 901-914.

Larwanou M., 2013. Espèces prioritaires et options agroforestières pour la mise en valeur de la Grande Muraille Verte. In : Dia A. \& Duponnois R., eds. Le projet majeur africain de La Grande Muraille Verte. IRD Éditions, 39-51.

Larwanou M. et al., 2010. Pratiques sylvicoles et culturales dans les parcs agroforestiers suivant un gradient pluviométrique nord-sud dans la région de Maradi au Niger. Tropicultura, 28(2), 115-122.
Lu C., Zhao T., Shi X. \& Cao S., 2018. Ecological restoration by afforestation may increase groundwater depth and create potentially large ecological and water opportunity costs in arid and semiarid China. J. Cleaner Prod., 176(1), 1213-1222.

Maes W.H. et al., 2009. Plant-water relationships and growth strategies of Jatropha curcas L. seedlings under different levels of drought stress. J. Arid Environ., 73(10), 877-884.

Mando A., Brussaard L. \& Stroosnijder L., 1999. Termiteand mulch-mediated rehabilitation of vegetation on crusted soil in West Africa. Restor. Ecol., 7(1), 33-41.

Maréchaux I. et al., 2015. Drought tolerance as predicted by leaf water potential at turgor loss point varies strongly across species within an Amazonian forest. Funct. Ecol., 29(10), 1268-1277.

Mefti M., Abdelguerfi A. \& Chebouti A., 2001. Étude de la tolérance à la sécheresse chez quelques populations de Medicago truncatula ( L .) Gaertn. In : Delgado I. \& Lloveras J., eds. Quality in lucerne and medics for animal production. Zaragoza, Espagne : CIHEAM, 173176.

Montwé D., Isaac-Renton M., Hamann A. \& Spiecker H., 2016. Drought tolerance and growth in populations of a wide-ranging tree species indicate climate change risks for the boreal north. Global Change Biol., 22(2), 806815 .

Nana R., Tamini Z. \& Sawadogo M., 2010. Effets d'un stress hydrique intervenu pendant le stade végétatif et la phase de floraison chez le gombo. Int. J. Biol. Chem. Sci., 3(5),1161-1170.

Otieno D.O., Schmidt M.W.T., Adiku S. \& Tenhunen J., 2005. Physiological and morphological responses to water stress in two Acacia species from contrasting habitats. Tree Physiol., 25(3), 361-371.

Reynolds J.F., Kemp P.R., Ogle K. \& Fernández R.J., 2004. Modifying the 'pulse-reserve' paradigm for deserts of North America: precipitation pulses, soil water, and plant responses. Oecologia, 141(2), 194-210.

SAS Institute Inc, 2015. JMP 12 Basic Analysis. New York. SER, 2002. The SER primer on ecological restoration, https://www.ntc.blm.gov/krc/uploads/399/ser_primer. pdf, (25 September 2019).

Zgallaï H., Steppe K. \& Lemeur R., 2007. Étude des caractères morphologiques des plantes de tomate soumises à un déficit hydrique en milieu hydroponique. Sécheresse, 18(1), 57-64.

(40 réf.) 